\title{
Example Analysis of Digital Wireless Mapping Applied to Construction Engineering Measurement
}

\author{
Pengfei Wang $(\mathbb{D}$, Yanli Wang, and Maohui Wang \\ Shandong Labor Vocational and Technical College, Jinan 250022, China \\ Correspondence should be addressed to Pengfei Wang; wangpengfei@sdlvtc.cn
}

Received 1 November 2021; Accepted 13 December 2021; Published 27 January 2022

Academic Editor: Gengxin Sun

Copyright (C) 2022 Pengfei Wang et al. This is an open access article distributed under the Creative Commons Attribution License, which permits unrestricted use, distribution, and reproduction in any medium, provided the original work is properly cited.

\begin{abstract}
This paper provides an in-depth study and analysis of the measurement of construction projects using digital wireless mapping and illustrates it using examples. Static measurements determine the relative positions between ground points with high accuracy using a baseline vector network composed of simultaneous observations by multiple receivers, using the correlation of errors in space and time. Technology-based on this error correlation, then the distance observation error or coordinate observation error measured by the base station is sent to the mobile station through the data chain to correct the station star distance observation or coordinate value of the mobile station, improving the operational accuracy and efficiency of the mobile station. The measurement avoids the tedious measurement organization work and long-time data collection and postcomputation work of static measurement, and the coordinates of the mobile station can be obtained in real-time. To verify whether the accuracy of the $3 \mathrm{D}$ model built by the $3 \mathrm{D}$ reconstruction method integrating UAV and camera (air ground) images can meet the accuracy requirements for the installation deviation detection of the assembled building components, the $3 \mathrm{D}$ reconstruction of the physical objects was completed by this method. The accuracy of the 3D model built by this method was evaluated by comparing the measurement coordinates of 25 prearranged checkpoints in the 3D model with those of the total station. The test results show that the maximum point accuracy of the $3 \mathrm{D}$ model is $2.305 \mathrm{~mm}$, and the average medium error is $2.147 \mathrm{~mm}$, which can meet the accuracy requirements of the installation deviation detection of the assembled building components. The detection of installation deviation of wooden columns was completed by measuring the 3D model, and it was verified that the method is intuitive, fast, accurate, and batch completion of the deviation detection of axis position, elevation, small labour, batch detection of components, and contactless mapping compared with the traditional detection methods.
\end{abstract}

\section{Introduction}

New instruments and technologies are needed to improve the traditional surveying and mapping technology to obtain spatial three-dimensional information. Unlike the traditional surveying and mapping measurement technology such as total station and GPS through a single point measurement way to collect data, three-dimensional laser scanning technology can obtain many point cloud data information, to obtain a more fine, complete information data of the object under test [1]. 3D laser scanning technology does not need to contact the measured object when measuring the target object, which can reach the number of hundred thousand points per second, and this efficiency of point collection is incomparable to traditional measurement technology [2]. Point cloud data can express the measured target object in more detail and accurately because of its huge amount of data and strong point density, but this also brings greater difficulties for data computing, processing, use, and storage [3]. A large amount of point cloud data requires high computer configuration, and it often leads to computational crashes and crashes when processing, which affects the efficiency of point cloud data processing and use, and then affects the efficiency of 3D model reconstruction of point cloud data, so point cloud data streamlining is especially important. It is of great significance for the application and development of 3D laser scanning technology and points cloud data to compress the data volume of point cloud data to the maximum extent while ensuring that the target object model features and reconstruction are not 
affected [4]. In turn, it affects the efficiency of point cloud data $3 \mathrm{D}$ model reconstruction, so point cloud data streamlining is particularly important. In this paper, we take the point cloud data of Nanjing subway station as experimental data, propose an improved method for the deficiency of not being able to retain the target area for point cloud data streamlining, and carry out the streamlining and compression of discrete point cloud data under the condition of avoiding the establishment of a topological relationship between points and points, which preserves the original format of point cloud data from being destroyed and provides a good data basis for the later processing and reconstruction of point cloud data.

The existing data exchange and storage management mode have obvious drawbacks, poor integration and management, and poor visualization. The monitoring of highspeed railway in operation period has the characteristics of long-term, complexity, and wide area, and there is less research on the monitoring data management of highspeed railway in operation period [5]. At the same time, the engineering department, measurement unit, evaluation unit, and the evaluation unit, as the hub to link all units, is an important link for data communication, data quality control, and progress control, while the evaluation system has not been systematically studied. Urban control measurement in urban surveying and mapping projects, and other work and control measurement, are mainly divided into two aspects of plane control measurement and elevation control measurement. Urban control survey is mainly divided into three stages of design, construction. The first is the establishment of the engineering control network and the mapping of the engineering topographic map [6]. These two kinds of measurements are mostly used in the preconstruction survey and design stage of urban construction. The second is the positioning of construction release. The last is the as-built measurement and deformation measurement, which are used for project quality inspection and later evaluation after the construction is finished.

The software system supporting them has been continuously improved, which makes the UAV tilt photogrammetry system have technical support and quality guarantee in various engineering applications. UAVs are widely used in many industries for their advantages such as good stability. Especially in the field of surveying and mapping, UAVs can acquire ground images with high efficiency and are used in many aspects such as drawing high precision medium and large-scale mapping and generating DEM (Digital Elevation Model) models. The various building components obscure each other, and it is difficult to use a single UAV to complete a full range of image data collection of the components, resulting in the lack of data on the obscured parts, holes, and other phenomena in the corresponding parts of the three-dimensional model established based on image data, affecting the measurement accuracy. The camera, with its advantages of free shooting angle and flexible operation, can provide an effective complement to the UAV image. Combining the characteristics and advantages of the UAV and camera, it can complete all-around and high-efficiency image data acquisition of the completed installation.

\section{Status of Research}

$3 \mathrm{D}$ reconstruction is an important method to obtain $3 \mathrm{D}$ information of an object. In recent years, the technical theory of $3 \mathrm{D}$ reconstruction and related software has been increasingly improved, and $3 \mathrm{D}$ reconstruction models of physical objects are widely used in mapping engineering, digital earth, urban planning, and other fields. The acquired point cloud data is subjected to a series of operations such as denoising, alignment, thinning, and encapsulation to realize the construction of a $3 \mathrm{D}$ point cloud model of the target object [7]; the 3D reconstruction method based on image data is to collect the image data of the object using unmanned aerial vehicles, cameras, and other equipment, based on the photogrammetry principle combined with relevant $3 \mathrm{D}$ reconstruction software to realize the automatic reconstruction work of the $3 \mathrm{D}$ model of the photographed object [8]. When using 3D laser scanners for all-around measurement of buildings, due to the restricted scanning angle of the instrument, it is often necessary to arrange multiple scanning sites, and the on-site data collection is timeconsuming; later, the point cloud data from different sites need to be spliced, and the amount of manual intervention is large; moreover, the amount of point cloud data is huge, and the processing and preservation costs of the data are high [9]. The image-based $3 \mathrm{D}$ reconstruction method is becoming more popular with the continued development of drones, cameras, and other equipment, the sensor accuracy continues to improve, and the corresponding technical theory and supporting software continues to improve, with its high-cost performance, high stability, good accuracy, and other advantages in the construction, mapping, and other engineering fields of application [10].

Point cloud data segmentation based on edges is investigated, and an algorithm for point cloud data segmentation using different judgments of point cloud data contours using information gradients and unit normal vector directions is proposed [11]. The original format of the point cloud data is not destroyed, providing a good data foundation for later point cloud data processing and reconstruction. An edgebased point cloud data segmentation method is proposed to use a three-dimensional moving boundary model for data segmentation to reduce the influence of noise in the judgment of point cloud data contours, but the computational efficiency is relatively slow. A region-based point cloud data segmentation study is conducted, proposing point cloud data segmentation based on the normal vector of points and their redundancy as a basis for region growth [12]. The second segmentation uses edge-based segmentation for point cloud data after the first segmentation, which improves the accuracy of the point cloud data segmentation algorithm [13]. The study of multiple point cloud data segmentation is carried out, based on edge and then facebased segmentation of point cloud data, first, the geodesic lines between different points in the point cloud data model are calculated, the contours formed by the segmentation lines are used as separation boundaries, and the point cloud data segmentation is carried out using region growing method [14]. 
The full building lifecycle is dynamic and includes the full cycle of design, production, construction, and operation and maintenance, up to demolition and recycling. In different stages of this process, the required spatial information of prefabricated components will be different according to specific project tasks, such as the outline dimensions of components in the component production stage, the timely access to transportation routes, vehicle locations, and yard unit locations in the transportation stage, the component positioning control points and lines in the construction and installation, and the floor and room numbers to which the components belong in the operation and maintenance stage, etc. In addition, many scholars consider component coding as an important element of component location information, which is the main basis for quickly identifying, locating, and managing target components from many similar prefabricated components in each project phase.

\section{Analysis of Digital Wireless Mapping Applied to Construction Engineering Measurement}

3.1. Digital Wireless Mapping Design for Construction Projects. The determination of the appropriate number of elevation and planimetric control points as the basis for topographic mapping is known as control surveying. In the case of plate meter mapping, there are two types of control surveys, namely, root and first-level control surveys. The latter is based on geodetic control points, combined with wire or triangulation methods, in the determination area of relatively uniform distribution, with a high degree of accuracy of the control points to determine; while the former is based on the first level of control, combined with small triangulation of fixed points to measure the way, the mapping needs of control points to encrypt to meet [15]. In addition, it can also be combined with the way of triangulation and elevation measurement to determine the elevation of the control point of the root of the map. Fragmentation measurement is a way of giving the topography as well as the mapping of the features. The terrain or feature points obtained during the mapping process are called fragmentation points. The location of the fractional point planes can be determined in conjunction with the polar method, and the elevation can also be determined by the rules of apparent distance measurement. As a hub for contacting various units, the evaluation unit is an important link in data communication, data quality control, and progress control, and the evaluation system has not yet been systematically studied. The mapping methods can be classified according to the type of instrument used, such as the latitude and longitude instrument and the plate meter mapping method. All the above methods have similar operational processes. Before the mapping work is carried out, mylar or drawing paper is fixed on the mapping board, the coordinate grid is drawn, the control points and contour points are spread, and the mapping operation is carried out after confirming that they are correct. In this process, the plotted or temporarily determined points are used as stations, the levelling plate is placed at the station and oriented, then the telescope is used to align the fragmented points, and the elevation and horizontal distance from the station to the fragmented points are determined in combination with the straight edge, and the edge length is intercepted along the straight edge in combination with the mapping scale, which is the plane position of the fragmented points on the map, and the elevation is marked. Thus, the topographic map is mapped by carrying out mapping activities at each station.

$$
\begin{gathered}
\left\{\begin{array}{l}
S=T\left(r^{2}\right), 0 \leq r \leq 1, \\
P_{s}(s)=P_{r}(\mathrm{r}) \frac{d r}{d s}, r>1,
\end{array}\right. \\
T(r)=\int_{0}^{T} \frac{P_{r}(\mathrm{r})}{d r} .
\end{gathered}
$$

The need to carry out the corresponding measurement work in the preconstruction preparation. This is to get the spatial location of each line to facilitate the construction of the project, especially in the construction process, it is necessary to restore the centreline of each pipeline in the design drawings to the reality of construction accurately. Let us take the construction of a railway as an example. When railway construction is carried out, several measurements are first taken, and the initial measurement data is obtained and then corrected to obtain accurate mapping data. In the preliminary surveying and mapping, the main task point is for the geographical condition along the way, and the mileage data and the corresponding pile numbers are obtained by calculation [16]. Two kinds of cross-sections are drawn out according to the illustrated elevation, and the corresponding engineering data are calculated to obtain a relatively feasible plan, which is then applied in the later construction. The socalled actual measurement is to implement the designed scheme in practice.

$$
\begin{gathered}
\frac{d T(r)}{d r}=\frac{d}{d r}\left[\int_{0}^{T} \frac{P_{r}(\mathrm{r})}{d r}\right] \\
{\left[\int_{0}^{T} \frac{P_{r}(\mathrm{r})}{d r}\right]=\frac{P_{r}(\mathrm{r})}{d r} \frac{3}{P_{r}\left(\mathrm{r}^{2}\right)} .}
\end{gathered}
$$

But the calculation process cannot be adjusted; parameters are not easy to control. In contrast, histogram matching can select the image with excellent effect as the matching object and correct the histogram of the original image to make it into a prescribed shape. Histogram correction and matching are particularly effective for low brightness image processing. When the sun altitude angle and light conditions in the same period and the same area are the same, the difference between the histogram distribution of the two images near is small. Conversely, there are large differences in the histograms of feature images taken by the camera when the time and external environment are different. In this paper, the target image to be corrected is radiometrically corrected according to the histogram of the reference image (the image with the most suitable image brightness and contrast are selected), so that the gray level interval of the target 
image and the reference image is the same, and the purpose of histogram correction is accomplished. Image matching is the process of finding homonymous points between two or more images, and image matching problems are generally solved using image correlation techniques in early research. In UAV photogrammetry, image matching technology is the key to automatically finding homonymous image points and generating orthophoto by null-three calculation, and the matching work can be carried out only through feature information, which mainly points feature, line feature, and area feature, among which, point feature extraction is the mainstream method for feature extraction due to its simple algorithm and low implementation difficulty, as shown in Figure 1. Due to its good stability and low cost, drones can complete large-scale and high-efficiency image data collection of ground objects, and the advantages of data timeliness and scientific have been widely used in many industries, especially in surveying and mapping. In the field, drones can obtain ground images efficiently.

Model light-weighting refers to the compression and extraction [17]. The methods for BIM data model lightweight can be broadly divided into two categories: internal lightweight and external lightweight of the model. Internal lightweight is mainly to delete the redundant information in the model, which usually includes information that is used infrequently, too detailed information, duplicated information, etc., to achieve the purpose of reducing the database [91]. A series of studies have been conducted in this area. They investigated lightweight methods based on component merging and discretization. Among them, in internal lightweight, by parsing the basic attribute information of the components, the corresponding attribute sets are extracted and the same attribute data are deleted; at the same time, the duplicate geometric representations in the model are deleted and a unified representation is established through the spatial location relationship to identify the components with the same geometric shape in the BIM model. In terms of internal lightweight, the main purpose is to identify the information in the O\&M phase and delete other unnecessary information (unnecessary information generated in the design and construction phases), which can be done by obtaining the set of data to be deleted through the provided filters and deleting them. It is difficult for a single UAV to collect all-round image data of the components, resulting in missing data in the occluded parts, and resulting in distortions and holes in the corresponding parts of the threedimensional model established based on the image data, which affects the measurement accuracy.

$$
\begin{aligned}
& V=A X+B t+L, \\
& t=A^{T} A+A^{T} B .
\end{aligned}
$$

It signals that the interpolated midpoint has shifted to its neighbouring loci, so the position of the currently significant point needs to be shifted. Also, interpolate multiple times on the new loci until convergence; perhaps exceeding the set frequency of iterations or exceeding the extent of the picture edges, when such points should be eliminated. In scale-space polar detection, because of the variable quality of polar points, there will inevitably be points among these polar points that do not satisfy the conditions. A poorly defined Gaussian difference algorithm has limits with great principal curvature at locations that span the boundary and little principal curvature in the direction of the vertical boundary, requiring the removal of fluctuating boundary response points, as shown in Figure 2.

The accuracy of digital results plays a decisive role in the feasibility of applying low-altitude photogrammetry to earth change monitoring. The accuracy analysis of digital results generally contains two aspects: theoretical accuracy and the real difference value of checkpoints. The calculation principle of theoretical accuracy is to regard the coordinate correction values of encrypted points as random errors, calculate the variance-covariance matrix of the correction numbers of coordinate points, and thus find out the levelling accuracy. The theoretical accuracy analysis reflects the error distribution law. The theoretical accuracy can be viewed in the levelling log under the project file. When the residuals are higher than 3 times the medium error, the point is treated as a rough point [18]. The true difference of checkpoint is compared by comparing the coordinate value of checkpoint with the field measurement value to find out the medium error of all checkpoints and control points. In general, this true difference value can reflect the real error more directly, and this method is also used in production for product quality analysis.

The amount of earth change is calculated from repeated measurements in a specific measurement area [19]. Therefore, its feasibility needs to consider not only the accuracy (precision) of measurement data but also the stability of each measurement data, both of which are indispensable, and the accuracy and stability of monitoring data depend on the absolute precision of single measurement and the relative precision of multiple measurements, respectively. The absolute accuracy of a single measurement can be obtained by checking the true difference of point coordinates, while the relative accuracy of multiple measurements is analysed by comparing data of multiple periods.

3.2. Design Analysis of Construction Engineering Measurement Examples. The 3D laser scanner acquires a huge amount of point cloud data of the target object, although a large amount of data provides very complete and detailed information of the target object, at the same time, it also brings great difficulties to the processing and application of the data, which is of great help to the application, processing, operation, and storage of point cloud data. Point cloud data streamlining and compression according to the data format are mainly divided into grid data and discrete data, based on the triangular grid streamlining is the first point of cloud data to establish the topological grid relationship to form triangular grid data, and then the triangular grid for data compression and deletion. The streamlining based on discrete point cloud data is to directly calculate the information of point cloud data and directly compress and delete the point cloud data according to the information. The streamlining based on point cloud data directly is more efficient, better, and more widely used, as 


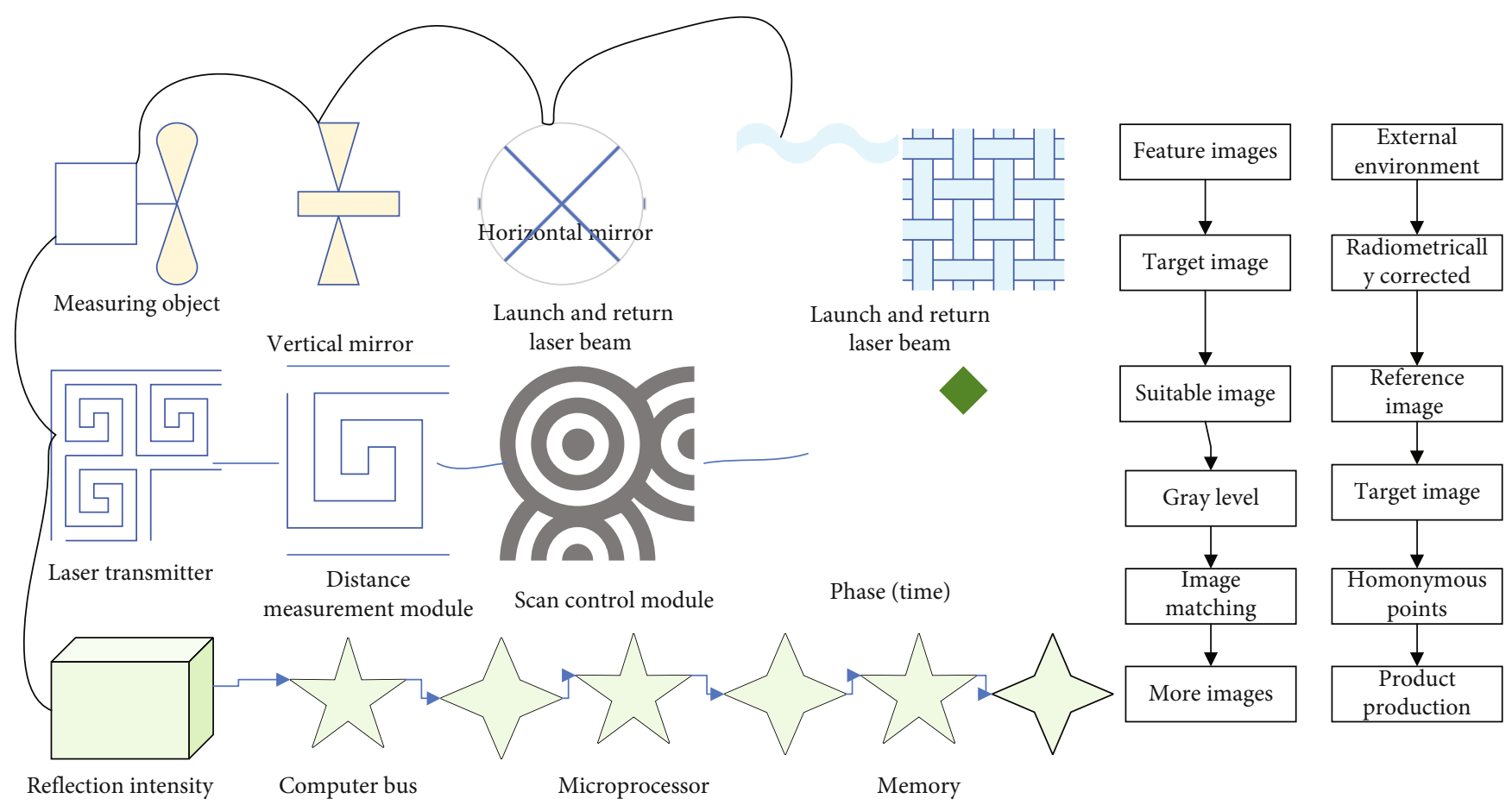

FIGURE 1: Working principle of 3D wireless mapping of buildings.

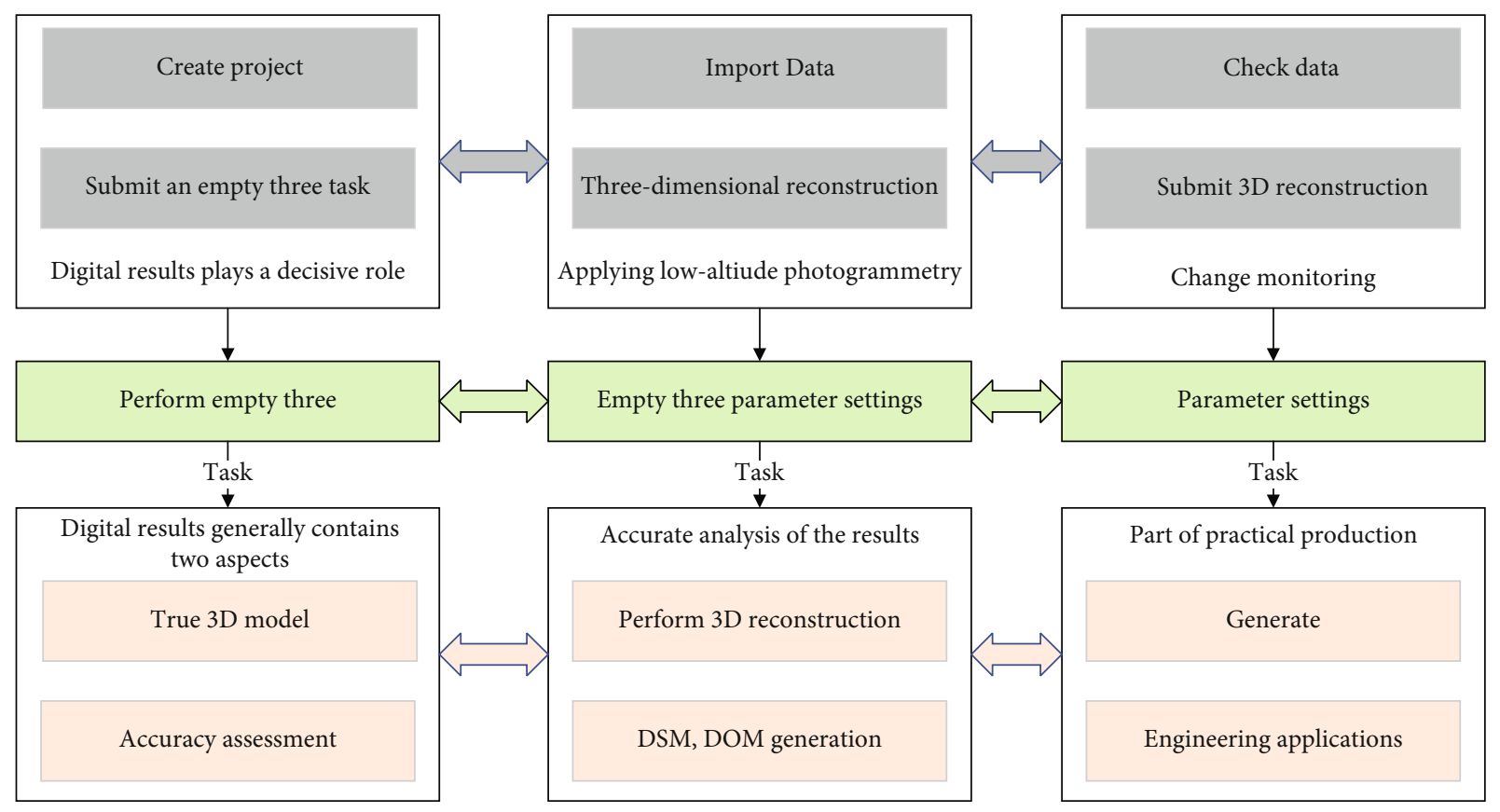

FIgURE 2: Data production process.

shown in Figure 3. Provide accurate data for the preliminary design. In the predesign, generally according to the position corresponding to the turning point, use the curve to connect the two adjacent straight lines to each other, according to the radius value given by the design and the steering angle obtained by the measurement, the element value of the curve is obtained through calculation.
The structural members of the assembled building should be positioned and installed following the modal grid, and the location of the datum (line) can be determined by the centreline positioning method, the interface positioning method, or the method of mixing the centreline and interface positioning methods. These three positioning methods have their characteristics and are suitable for different 


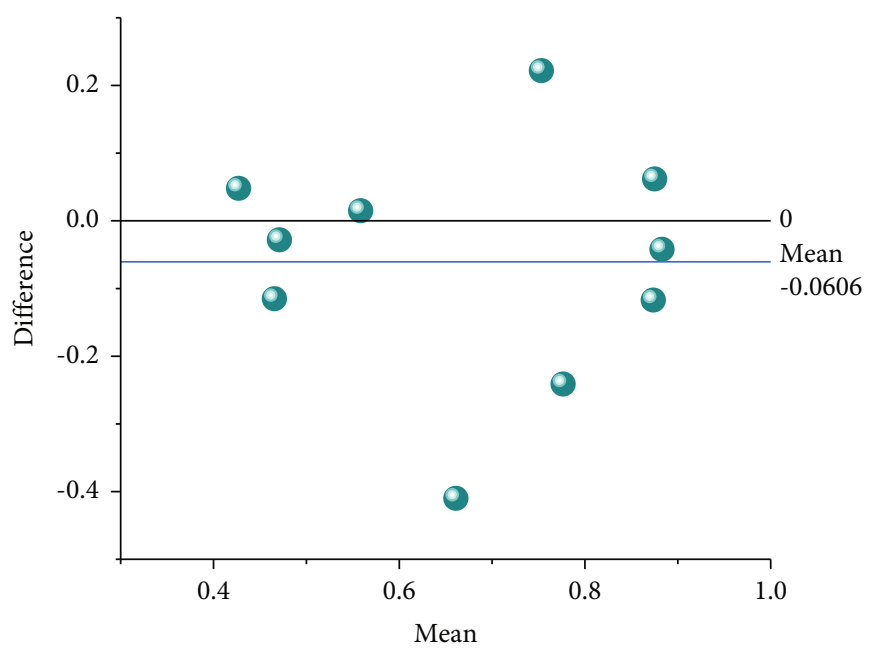

Figure 3: Building modulus coordination criteria.

component positioning and modulus grid space requirements. The centre positioning method is to coincide the positioning datum (line) with the physical centre of the member. This method is beneficial to the prefabrication, positioning, and installation of the member, so when the structural members are not connected adjacent to other members, the centre positioning method can generally be used, beams, and load-bearing walls. However, when the centre positioning method is used for the main structural members, it may cause the nonmodularity of the interior decoration space, which is not convenient for the setting of the decoration space grid and the positioning and installation of the decoration members, and the modal decoration space needs to be formed by adjusting the thickness of the walls. The use of the interface positioning method can make the interface of the members overlap with the positioning datum (line) to avoid the unevenness of the space interface or the formation of nonmodular space due to the different sizes of structural members and space dividing members. When the structural members are installed continuously, the interface of the previous member is the installation datum of the next member, the members along a certain interface need to be installed completely flat, and the interface positioning method should be used, such as the positioning of floor slabs and roofs. Make the gray level interval of the target image and the reference image the same, and achieve the purpose of completing the histogram correction. Image matching is a process of finding points with the same name between two or more images. In early research, image-related technologies are generally used to solve image matching problems. In practical application, only one positioning method often cannot meet the requirements of construction, such as the main structural member positioning and installation requirements at the same time to meet the datum surface positioning, or the main structure wall installation thickness needs to meet the modulus size, often use the method of centre positioning axis, interface positioning line superimposed on the same modulus grid.

The deepening design of the assembled building refers to the construction drawings with implement ability based on the original design scheme and condition drawings, should complete the design of a flat and vertical section of the building, the design of cross-section and reinforcement of structural members, the designers should refine the parameters of the components and determine reasonable production and installation tolerances according to the comprehensive requirements of various professions and project links, such as architecture, structure, and equipment, and the content and depth should meet the requirements of component processing, as shown in Figure 4.

When only orthophoto is used in the modelling, the elevation accuracy is gradually improved with the encryption of image control points, but the improvement effect is gradually weakened, and the medium error in the elevation of image control points is the same in scheme e and scheme $\mathrm{f}$, both of which is $0.014 \mathrm{~m}$, and the medium error in the elevation of checkpoints is $0.029 \mathrm{~m}$ and $0.023 \mathrm{~m}$, respectively, which is not much different. This indicates that increasing the density of the image control points can improve the accuracy of the null-three solution, but it is not the case that the greater the density of control points is better, and a reasonable layout plan and number of image control points should be chosen according to the project requirements [20]. Theoretically, the weakest point of the model should be around the survey area, and the error control around the survey area should be mainly considered when setting up the image control points, so the mid-error of the elevation is $0.067 \mathrm{~m}$ when using scheme $\mathrm{c}$, i.e., the image control points are evenly set up around the area, which is a significant improvement in accuracy compared with schemes a and $b$. However, in the actual modelling, if the survey area is not evenly set up, the accuracy of the model can be improved. Calculate the variance-covariance matrix of the correction number of the coordinate point to find the accuracy of the adjustment. Theoretical accuracy analysis reflects the law of error distribution, and the theoretical accuracy can be viewed in the adjustment log under the engineering file. However, in the actual modelling, if the elevation error in some areas inside the survey area is still high, adding internal control points can effectively control the internal 


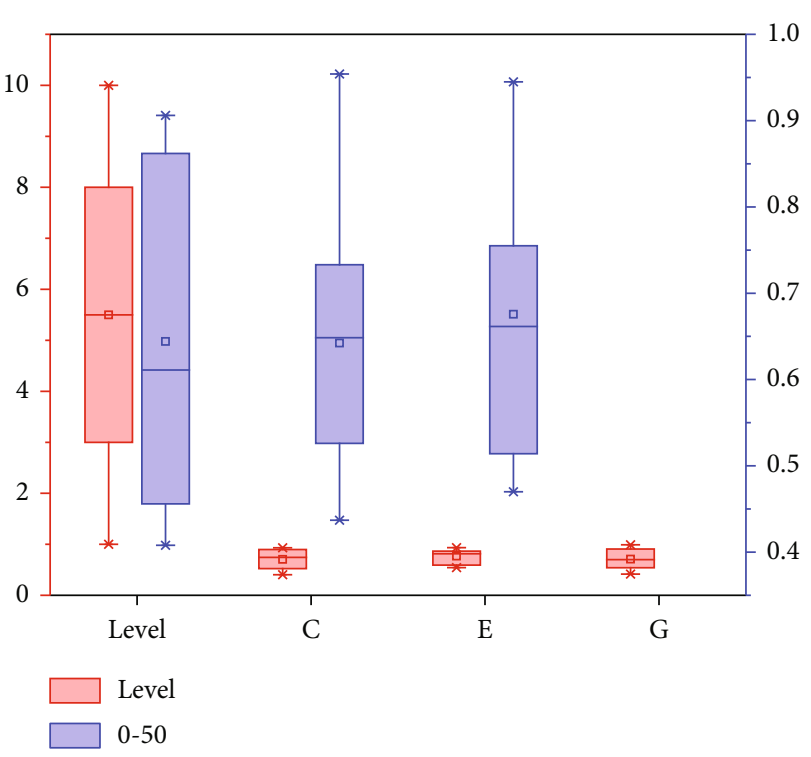

Figure 4: Elevation errors for different scenarios.

accuracy. When the tilted image is included in the modelling and option a is used, i.e., the single point layout at the four corners, the mid-elevation error of the control point is $0.018 \mathrm{~m}$ and the mid-elevation error of the checkpoint is $0.026 \mathrm{~m}$, which is slightly worse than the mid-elevation errors of $0.014 \mathrm{~m}$ and $0.023 \mathrm{~m}$ when only the orthophoto and option $\mathrm{f}$ is used in the modelling, and the maximum residual value of both options is $3 \mathrm{~cm}$. This indicates that the inclusion of tilted images in the modelling has an obvious binding effect on the model elevation error.

As we all know, before collecting image data for the first time, we need to use GNSS-RTK to measure the coordinates of image control points for internal processing, and the coordinates of the first measured image control points are also used in the later processing of multiphase data, but in actual engineering, the previously laid out image control points are often destroyed, and if the image control points are relaid, it will affect the operation efficiency and the accuracy of data processing. In the calculation of multiperiod DSM data overlay, the sampling points with elevation differences less than $1 \mathrm{~cm}$ can be selected as relatively stable image control points for subsequent internal modelling, which can effectively reduce the inconvenience caused by image control points remeasurement and improve the relative elevation accuracy of DSM overlay.

\section{Results and Analysis}

4.1. Digital Wireless Mapping Performance of Construction Projects. The image data captured by the UAV gimbal camera is first stored on a memory card carried by the camera, while the wireless image transfer module is used to transfer the captured image data back to the staging database of the automated airport system. The camera's memory card has limited memory, and the memory card is cleared after the mission to make sure that the data has been completely returned to the staging database. The staging database has much more memory than the memory card carried by the camera, but also has limited storage space and will also be cleared after multiple phases of data collection to ensure that the data has been fully uploaded to the backend cloud database. It plays a great role in the measurement of historical sites, cultural relics and historical sites restoration, archaeological site reproduction simulation, cultural relics and historical sites data storage, and large-scale historical site surveying and mapping. The image data collected by the UAV gimbal camera and other process data of the system will eventually be uploaded to the cloud database for professional staff to download and process. The cloud database should have a large storage space, and through the continuous accumulation of data, it will eventually form engineering monitoring big data and provide the possibility for big data analysis. The automatic skylight realizes the function of automatic opening and closing of the UAV before and after take-off and before and after landing, using a stepper motor and a limit switch to control the movement of the skylight. The stepper motor drives the skylight movement through gears and chains, and the limit switch controls the closing motor according to the position of the skylight movement to complete the task of opening or closing the skylight, as shown in Figure 5.

Each point has 2 groups of data acquisition, each group with a different instrument height, more than 15 data acquisition, each data acquisition time of 3 seconds, two groups of data count 30 data through the levelling calculation, you can get the point after the levelling correction of the coordinate value. After the collection, you can use the instrument to do the preliminary calculation in the field and check whether the data is acceptable. If not, collect one or two more sets until you can pass the calculation. Ideally, the data collection time for each point is about 2 minutes. Adding the time of erecting stations, walking between points, and waiting for the fixed solution of the instrument between groups, it takes about 20 minutes at most, and 480 minutes for 8 hours of work a day, which can collect more than 24 points a day it took 3 days to complete the field control measurement data collection of 65 points.

In the actual measurement, the orientation is carried out with disk-left and disk-right observations, resulting in observation data, which are checked by the software site to see if the observation data exceeds the limits and if there are errors in the control point spacing. If the orientation is correct, then the data collection of pipeline points is carried out. When the pipeline point data is measured, the attribute data of each pipeline point can be input directly on the EPS software, and the connection relationship of the pipeline point can be outlined in the field so that the graph can be directly compared with the current situation, and errors such as connection and flow direction can be found and solved in the field. It is necessary to form a modular decoration space by adjusting the thickness of the wall. Using the interface positioning method can make the interface of the component coincide with the positioning reference plane (line) and avoid the unevenness of the space interface or the formation of a non-modular space due to the different sizes of structural components and space division components. The 


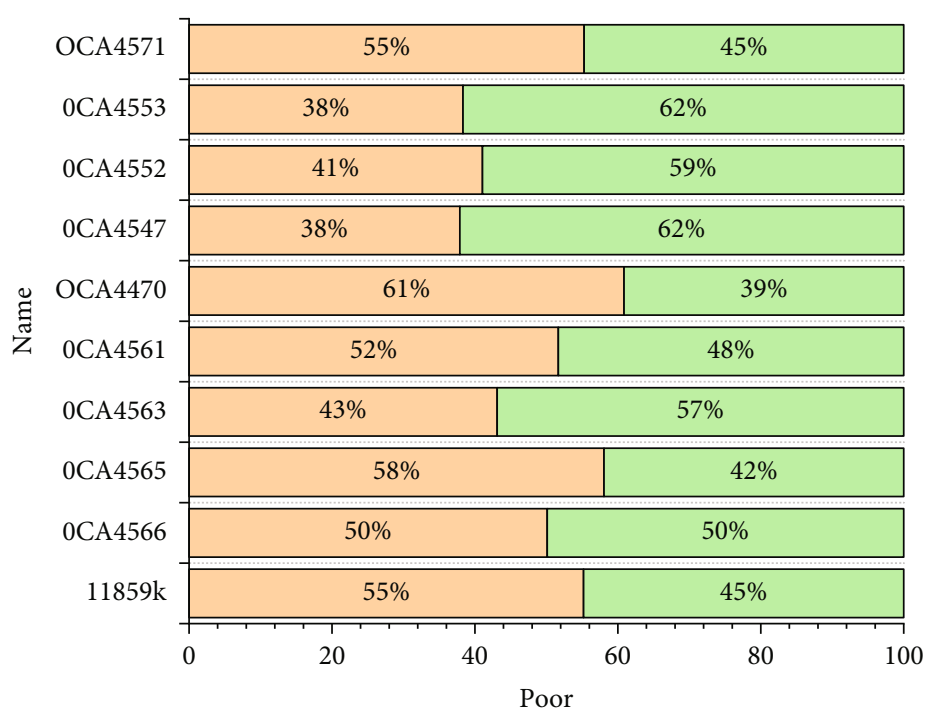

Elevation

Flat

Figure 5: Accuracy of the observed data.

pipeline diagrams, which have been measured and initially edited externally, are imported into the EPS software on the PC for further editing and finishing. Add markups and solve problems such as landing on the drawing surface. After self-checking and checking and modifying by the quality inspector, the final topographic pipeline result map was formed as shown in Figure 6.

The landing deviation is mainly concentrated around $10 \mathrm{~cm}$ at wind level 1 , within $20 \mathrm{~cm}$, at wind level 3 , and within $30 \mathrm{~cm}$ at wind level 4 . In wind level 4 , the landing deviation was around $30 \mathrm{~cm}$, and in wind level 5, only a small portion of the landing deviation was greater than $40 \mathrm{~cm}$. This indicates that the M100 drone achieves good landing accuracy in this simulation environment, and the landing error has certain randomness as the wind speed increases. The landing deviation of the drone is related to its ability to land safely and accurately on the designed automatic landing pad, which is a prerequisite for the monitoring system to realize the functions of collection and autocontinuation. Therefore, the simulation experiments initially verify the technical feasibility of the monitoring system in the absence of the M100 real aircraft.

\section{Design Results of Construction Engineering Measurement Examples}

UAV automatic landing pad, communication, and data management and processing are designed; the functional layers of the system and their interlogical relationships are elaborated; the automatic landing pad of the system, including the general structure of the box-type dock and the automatic range function settings. Meanwhile, considering the limitation of weather conditions on the unattended UAV operation mode, the composition and functional design of the environmental sensing system were discussed, and the

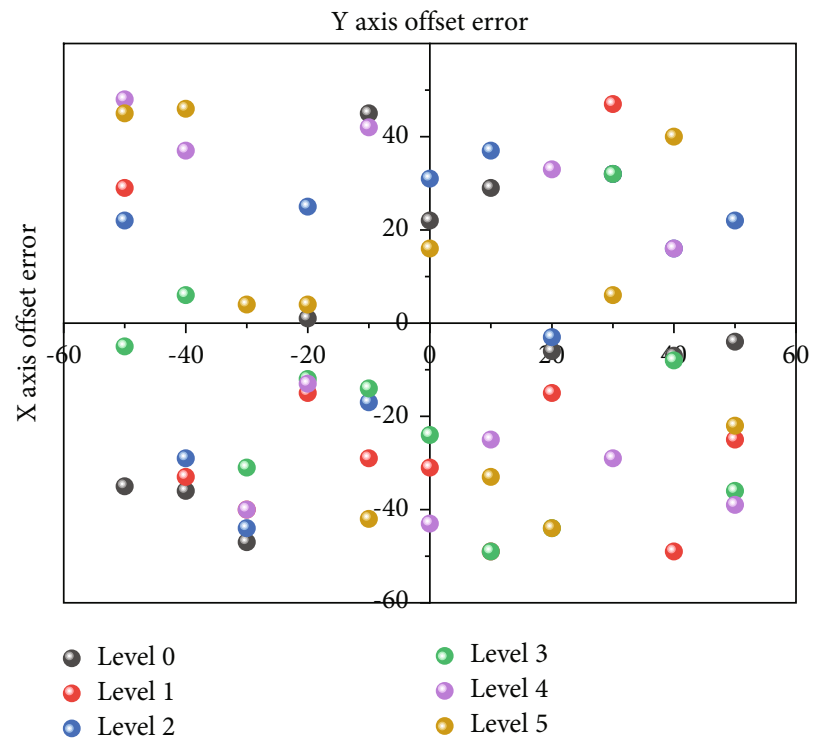

FIgURE 6: Offset error.

detailed operation process of the UAV and the automatic landing pad was determined. Finally, the technical feasibility of the unattended UAV monitoring system was initially verified by simulating the landing process and landing accuracy of the UAV and comparing it with the actual landing deviation of DJI Genie.

The first type is the splicing target, which is mainly used to increase the accuracy of automatic splicing because of the automatic splicing operation by the professional point cloud processing software, and the additional targets are mainly needed in the case of difficult viewing conditions and little information transmission between two adjacent stations, which is deployed according to the coordinate alignment 


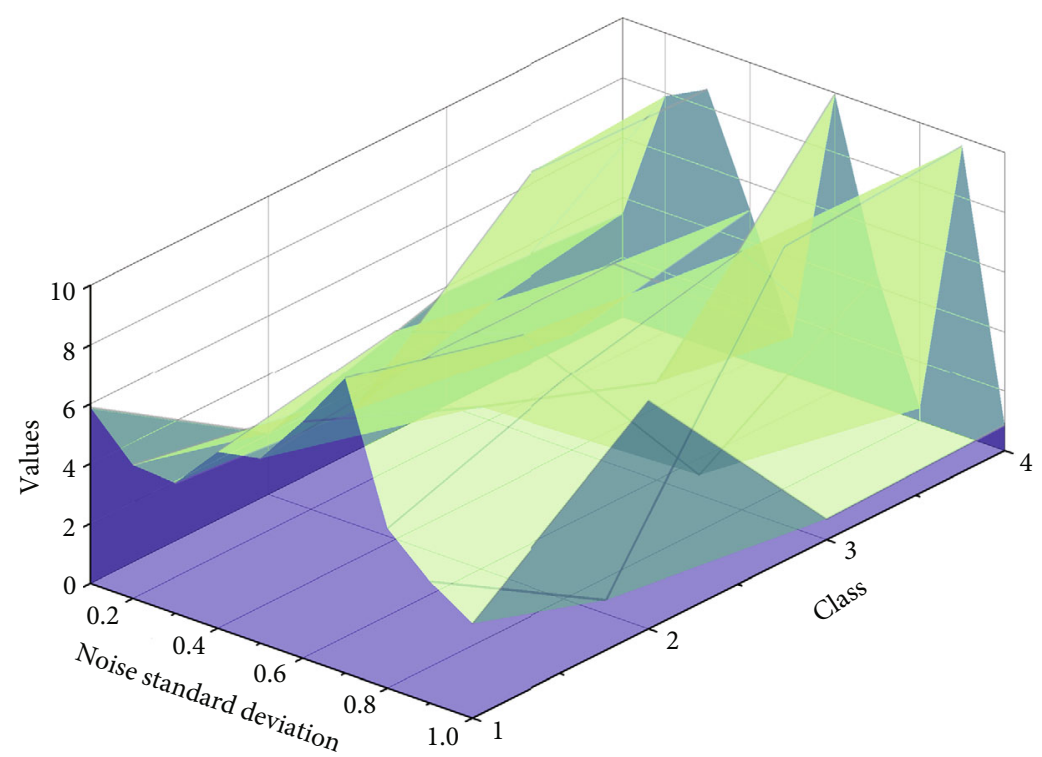

FIGURE 7: Rotational transformation parameter error.

requirements, which is deployed according to the coordinate alignment requirements, usually at the entrance and exit of the subway station or at the ground or underground level. After setting up the instrument, insert the battery, open the protective cover of the storage disk, insert the storage disk, the scanner starts to scan and rotate with itself, after one week of rotating and scanning, the scanner stops and stands still, indicating the completion of the current station, turn off the instrument and move to the next station to collect and scan, as shown in Figure 7.

Under the same noise standard deviation condition, the quaternion method outperforms the singular value decomposition method for the solution of the rotation transformation parameters. As the noise standard deviation keeps increasing, the error of the rotation parameters solved by both algorithms increases, and the error of the rotation parameters solved by the indicated quaternion is consistently lower than the error of the rotation parameters solved by the indicated singular value decomposition method. It shows that the accuracy of the solution of the quaternion method is higher than that of the singular value decomposition method in the solution of the rotation transformation parameters under the same noise conditions. Point cloud data alignment, also known as point cloud data stitching. The target object structure is complex, the target object exists between the occlusion and the limited view angle of the instrument itself, so in a single station set up, 3D laser scanner cannot collect the complete target object point cloud data and need to carry out multistation point cloud data scanning. Since the point cloud data of multiple stations are collected under separate coordinate systems, the point cloud data of multiple stations need to be stitched into one coordinate system, which requires point cloud data alignment processing, as shown in Table 1.

The average error of the $x$-axis is $0.0121 \mathrm{~m}, y$-axis is $0.0105 \mathrm{~m}$, and $z$-axis is $0.0159 \mathrm{~m}$. The overall error in each coordinate direction is no more than $0.02 \mathrm{~m}$, and the maxi-
TABLE 1: Comparison of coordinates of target points.

\begin{tabular}{lcccc}
\hline Point name & $X$ & $Y$ & $Z$ & Error \\
\hline$Z 1$ & 2.5 & 4.8 & 4.7 & 3.4 \\
$Z 2$ & 4.2 & 2.2 & 2.1 & 4.1 \\
$Z 3$ & 3.1 & 4.9 & 3.2 & 4.8 \\
$Z 4$ & 2.7 & 2.4 & 3.4 & 3.9 \\
$Z 5$ & 4.6 & 2.4 & 4.8 & 3.1 \\
$Z 6$ & 4.2 & 4 & 4.6 & 3.9 \\
\hline
\end{tabular}

mum error in the $z$-axis direction is $0.0159 \mathrm{~m}$, because the subway station is divided into a negative one and negative two layers, and in the point cloud data. This is because the subway station is divided into two layers: negative one and negative two, and the error is increased due to the splicing between different layers when the point cloud data is spliced.

Point cloud data unification is a way to optimize the overall point cloud data, to unify the overall point cloud of multiple stations after stitching into the overall point cloud data of a single station, and after the point, cloud unification process is completed, the operation of point cloud data selection and browsing becomes smoother on the original basis. In the process of point cloud data unification, point cloud data thinning parameters can be set according to the needs of modelling and point cloud data application to adjust the density and size of point cloud data.

\section{Conclusion}

The requirements of urban surveying on measurement technology are increasing, the operation environment of urban surveying is complex and changeable, due to the influence of visibility and operation conditions, the traditional measurement operation mode can no longer well meet the needs of urban surveying, TREK technology can be widely used in 
many fields of urban surveying because of its flexible and convenient work and fewer operation conditions, but its reliability is worse than the conventional static relative measurement. Some, so in using CORES technology measurement especially in control measurement operations, it is necessary to conduct repeat measurements appropriately and consider using different observation instruments and distributing repeat measurements at different observation times to improve the reliability of CORES measurements. There is no obvious systematic error, but the plane error leads to a large relative error in elevation in areas with abrupt elevation changes such as the edges of buildings and the edges of pits. By comparing the distribution and number of point clouds with elevation differences in different intervals, it was found that the number of high-precision sampling points with elevation differences less than $0.01 \mathrm{~m}$ in three groups accounted for $21.99 \%, 21.5 \%$, and $23.3 \%$ of the total, respectively. This can improve the relative accuracy of multiphase image modelling and reduce the negative impact caused by the destruction of the resurvey of image control points.

\section{Data Availability}

The data used to support the findings of this study are available from the corresponding author upon request.

\section{Conflicts of Interest}

The authors declare that they have no known competing financial interests or personal relationships that could have appeared to influence the work reported in this paper.

\section{References}

[1] M. Mahmood, D. Mzurikwao, Y. S. Kim et al., "Fully portable and wireless universal brain-machine interfaces enabled by flexible scalp electronics and deep learning algorithm," Nature Machine Intelligence, vol. 1, no. 9, pp. 412-422, 2019.

[2] S. Bi, J. Lyu, Z. Ding, and R. Zhang, "Engineering radio maps for wireless resource management," IEEE Wireless Communications, vol. 26, no. 2, pp. 133-141, 2019.

[3] R. Ponuma and R. Amutha, "Compressive sensing based image compression-encryption using novel 1D-chaotic map," Multimedia Tools and Applications, vol. 77, no. 15, pp. 19209-19234, 2018.

[4] A. Rashidi, M. Maghiar, and M. H. Sigari, "Capturing geometry for labeling and mapping built infrastructure: an overview of technologies," Iranian Journal of Science and Technology, Transactions of Civil Engineering, vol. 41, no. 4, pp. 415-428, 2017.

[5] T. R. Ray, J. Choi, A. J. Bandodkar et al., "Bio-integrated wearable systems: a comprehensive review," Chemical Reviews, vol. 119, no. 8, pp. 5461-5533, 2019.

[6] Z. Ma, J. Xie, H. Li et al., "The role of data analysis in the development of intelligent energy networks," IEEE Network, vol. 31, no. 5, pp. 88-95, 2017.

[7] K. Lee, X. Ni, J. Y. Lee et al., "Mechano-acoustic sensing of physiological processes and body motions via a soft wireless device placed at the suprasternal notch," Nature Biomedical Engineering, vol. 4, no. 2, pp. 148-158, 2020.

[8] S. M. Sepasgozar, H. Li, S. Shirowzhan, and V. W. Tam, "Methods for monitoring construction off-road vehicle emissions: a critical review for identifying deficiencies and directions," Environmental Science and Pollution Research, vol. 26, no. 16, pp. 15779-15794, 2019.

[9] M. Shafiei Dizaji, M. Alipour, and D. K. Harris, "Leveraging full-field measurement from 3D digital image correlation for structural identification," Experimental Mechanics, vol. 58, no. 7, pp. 1049-1066, 2018.

[10] C. Z. Dong and F. N. Catbas, "A review of computer visionbased structural health monitoring at local and global levels," Structural Health Monitoring, vol. 20, no. 2, pp. 692-743, 2021.

[11] S. M. Won, H. Wang, B. H. Kim et al., "Multimodal sensing with a three-dimensional piezoresistive structure," ACS Nano, vol. 13, no. 10, pp. 10972-10979, 2019.

[12] A. Buffi, A. Michel, P. Nepa, and B. Tellini, "RSSI measurements for RFID tag classification in smart storage systems," IEEE Transactions on Instrumentation and Measurement, vol. 67, no. 4, pp. 894-904, 2018.

[13] E. van der Kruk and M. M. Reijne, "Accuracy of human motion capture systems for sport applications; state-of-theart review," European Journal of Sport Science, vol. 18, no. 6, pp. 806-819, 2018.

[14] M. Carminati, O. Kanoun, S. L. Ullo, and S. Marcuccio, "Prospects of distributed wireless sensor networks for urban environmental monitoring," IEEE Aerospace and Electronic Systems Magazine, vol. 34, no. 6, pp. 44-52, 2019.

[15] J. Koo, M. R. MacEwan, S. K. Kang et al., "Wireless bioresorbable electronic system enables sustained nonpharmacological neuroregenerative therapy," Nature Medicine, vol. 24, no. 12, pp. 1830-1836, 2018.

[16] A. K. Srivastava, A. Dev, and S. Karmakar, "Nanosensors and nanobiosensors in food and agriculture," Environmental Chemistry Letters, vol. 16, no. 1, pp. 161-182, 2018.

[17] T. Kokulnathan, T. Sakthi Priya, and T. J. Wang, "Surface engineering three-dimensional flowerlike cerium vanadate nanostructures used as electrocatalysts: real time monitoring of clioquinol in biological samples," ACS Sustainable Chemistry \& Engineering, vol. 7, no. 19, pp. 16121-16130, 2019.

[18] E. Bakhtavar, M. Valipour, S. Yousefi, R. Sadiq, and K. Hewage, "Fuzzy cognitive maps in systems risk analysis: a comprehensive review," Complex \& Intelligent Systems, vol. 7, no. 2, pp. 621-637, 2021.

[19] J. Y. Dai, W. Tang, L. X. Yang et al., "Realization of multimodulation schemes for wireless communication by timedomain digital coding metasurface," IEEE Transactions on Antennas and Propagation, vol. 68, no. 3, pp. 1618-1627, 2020.

[20] S. Wolny, A. Mazak, C. Carpella, V. Geist, and M. Wimmer, "Thirteen years of SysML: a systematic mapping study," Software and Systems Modeling, vol. 19, no. 1, pp. 111-169, 2020. 